\title{
Ultrasound-guided femoral and popliteal sciatic nerve blocks for below knee surgery in patients with severe cardiac disease
}

\author{
Yun Suk Choi ${ }^{1}$, Hyeon Ju Shin², Ji-Yong Park ${ }^{2}$, Hyun Jung Kim ${ }^{1}$, and \\ So-Hui Yun ${ }^{1}$ \\ Department of Anesthesiology and Pain Medicine, ${ }^{1}$ Jeju National University School of Medicine, Jeju, \\ ${ }^{2}$ Anam Hospital, Korea University College of Medicine, Seoul, Korea
}

Patients with dilated cardiomyopathy or acute myocardial infarction are at risk of anesthesia complications, and peripheral nerve blocks (PNBs) provide more stable operative hemodynamics than general anesthesia or central neuraxial blocks. We present two rare cases of patients with dilated cardiomyopathy or acute myocardial infarction and a coagulation abnormality that were administered ultrasound-guided femoral and popliteal sciatic nerve blocks as anesthesia for lower limb surgery.

A 71-year-old woman (155 cm, $44 \mathrm{~kg}$, ASA 4) was scheduled to undergo open reduction and internal fixation due to a left lateral malleolus fracture. Five year previously, she underwent cardioversion and atrial ablation due to atrial fibrillation and ventricular tachycardia, and was being medicated for liver cirrhosis and dilated cardiomyopathy. Preoperative 2-dimensional electrocardiography showed atrial fibrillation and a left ventricular ejection fraction of 20-25\%. Her pulmonary arterial pressure was $30 \mathrm{mmHg}$. Clopidogrel (75 mg) was maintained during the perioperative period to prevent a thromboembolic event. Prothrombin time international normalized ratio (PT INR) was 1.36, prothrombin time (\%) was $64 \%$, and aPTT was $36.6 \mathrm{sec}$. Ultrasound-guided femoral and popliteal sciatic nerve blocks were chosen due to the risks posed by general anesthesia and a central neuraxial blockade. For the femoral nerve blockade,

Corresponding author: Hyeon Ju Shin, M.D., Ph.D.

Department of Anesthesiology and Pain Medicine, Anam Hospital, Korea University College of Medicine, 73, Inchon-ro, Seongbuk-gu, Seoul 02841, Korea

Tel: 82-2-920-5632, Fax: 82-2-929-2936

E-mail:may335@naver.com

Korean J Anesthesiol 2015 October 68(5): 513-515 http://dx.doi.org/10.4097/kjae.2015.68.5.513 the patient was placed in the supine position and a linear 6-13 MHz probe (SonoSite M-Turbo ${ }^{\circledR}$, SonoSite Bothell, WA, USA) was positioned at left inguinal crease level. Subsequently, after penetrating the fascia iliaca, a 22-gauge $50 \mathrm{~mm}$ insulated needle was inserted using the in-plane approach near the femoral nerve (Fig. 1A). After obtaining quadriceps femoris muscle contraction at $0.5 \mathrm{~mA}$ and negative blood aspiration, $20 \mathrm{ml}$ of $0.5 \%$ ropivacaine containing $1: 200,000$ epinephrine was injected. For the popliteal sciatic nerve blockade, the patient was placed in the right lateral decubitus position, and the same type of probe was placed at $3 \mathrm{~cm}$ above the popliteal crease level and a 22-gauge 50 $\mathrm{mm}$ long insulated needle was inserted near the popliteal sciatic nerve (Fig. 1B). After obtaining plantarflexion of the foot at 0.5 $\mathrm{mA}$ and negative blood aspiration, $20 \mathrm{ml}$ of $0.5 \%$ ropivacaine containing $1: 200,000$ epinephrine was injected. Twenty minutes later, loss of cold sensation in the surgical area and motor paralysis of the foot were confirmed and surgery was started. The nerve block took 23 minutes to perform and the surgery took 35 minutes.

The other case involved a 63-year-old woman $(155 \mathrm{~cm}, 67 \mathrm{~kg}$, ASA 4), who underwent below knee amputation due to thrombotic occlusions of the right femoral and popliteal arteries. She suffered from hypertension and rheumatoid arthritis. Three days prior to surgery, acute myocardial infarction had been diagnosed. Coronary angiography showed complete occlusion of the right coronary artery, which was resolved by percutaneous coronary intervention. Preoperative chest radiography indicated cardiac hypertrophy and pulmonary congestion. Heparin (7000+5000 $\mathrm{u}$ ) and clopidogrel (75 mg) were maintained during perioperative period to prevent a thromboembolic event. Coagulation test results yielded PT INR 1.45, prothrombin time 46\%, PTsec 15.6

(c) This is an open-access article distributed under the terms of the Creative Commons Attribution Non-Commercial License (http://creativecommons.org/ licenses/by-nc/4.0/), which permits unrestricted non-commercial use, distribution, and reproduction in any medium, provided the original work is properly cited. 

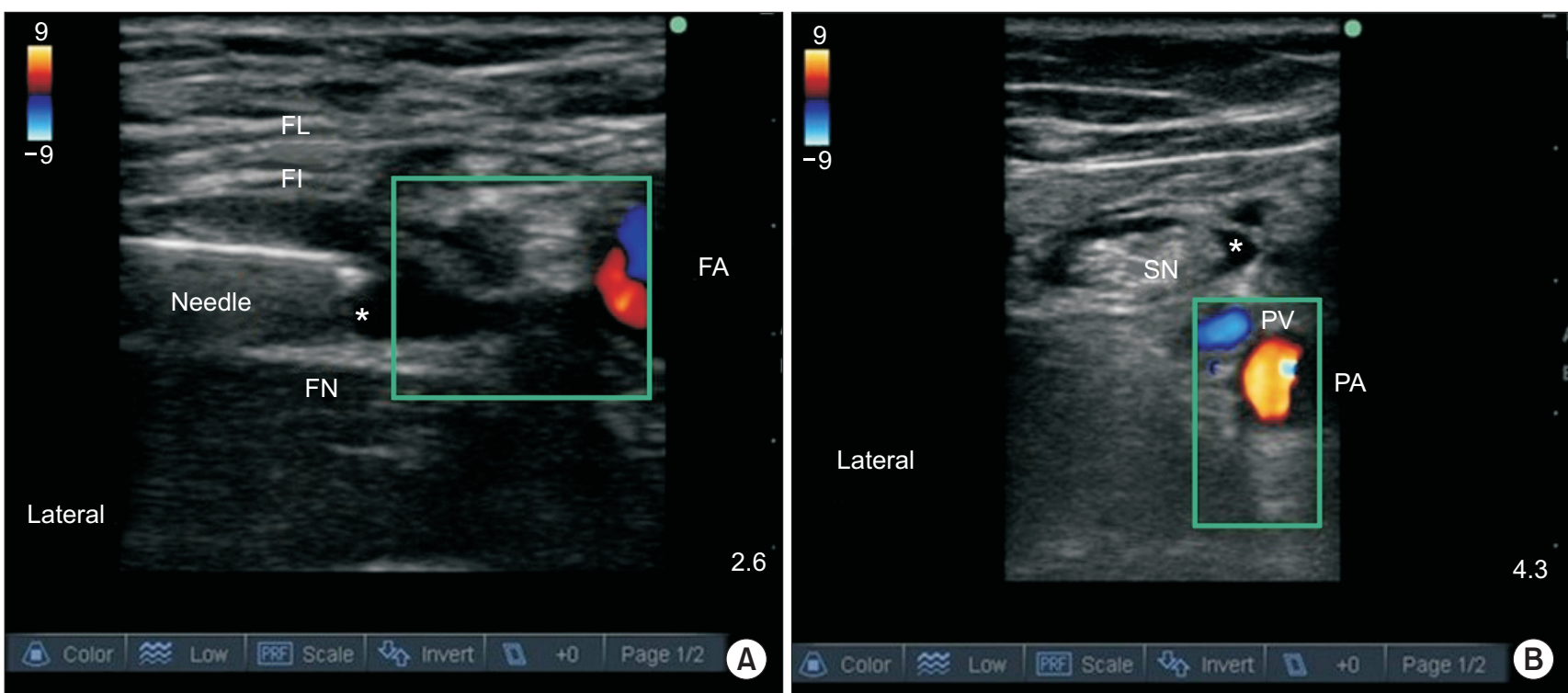

Fig. 1. (A) Ultrasound image of the femoral nerve block. FA: femoral artery, FN: femoral nerve, FL: fascia lata, FI: fascia iliaca, *local anesthetic. (B) Ultrasound image of the sciatic nerve block. The local anesthetic was injected circumferentially around the nerve. PA: popliteal artery, PV: popliteal vein, SN: sciatic nerve, *local anesthetic.

sec, and aPTT $44 \mathrm{sec}$. The patient also exhibited symptoms of septic shock resulting from hypotension and severe metabolic acidosis (pH: 7.29, BE: $-13.3 \mathrm{mmol} / \mathrm{L}$ ) resulting from hypoxic tissue damage due to myocardial infarction and disturbance of lower leg circulation. The patient was transfer to the OR, she was administered dopamine $(40 \mu \mathrm{g} / \mathrm{kg} / \mathrm{min})$, dobutamine $(20 \mu \mathrm{g} / \mathrm{kg} /$ $\mathrm{min})$, norepinephrine $(74 \mu \mathrm{g} / \mathrm{min})$, and hydrocortisol $50 \mathrm{mg} /$ day. The patient had a moon face and found it difficult to open her mouth sufficiently for to enable endotracheal intubation, and thus, for this reason and the presence of hemodynamic instability, ultrasound-guided combined femoral and sciatic nerve blocks were chosen. Upon arrival at the operation room, oxygen was supplied through a nasal cannula at $3 \mathrm{~L} / \mathrm{min}$, and the patient was sedated with $25 \mu \mathrm{g}$ of fentanyl and $1 \mathrm{mg}$ of midazolam. For the femoral nerve blockade, after negative blood aspiration after obtaining of the contraction of the quadriceps femoris muscle at $0.5 \mathrm{~mA}, 10 \mathrm{ml}$ of $0.75 \%$ ropivacaine containing epinephrine $1: 100,000$ was injected near the femoral nerve. For the popliteal sciatic nerve blockade, after negative blood aspiration and obtaining of plantarflexion and dorsiflexion of the foot at $0.5 \mathrm{~mA}$, $20 \mathrm{ml}$ of $0.75 \%$ ropivacaine containing epinephrine $1: 100,000$ was injected. Twenty minutes later, loss of cold sensation and motor paralysis of the surgical area were confirmed, and surgery was started. During surgery, midazolam $2 \mathrm{mg}$ and fentanyl 50 $\mu \mathrm{g}$ were injected for sedation and pain control. In this case, the nerve block took 25 minutes and the surgery 52 minutes. No cardiovascular complications were encountered during the peri- operative period and no femoral or popliteal artery hematoma developed over 14 days of follow-up in either case.

Patients with cardiomyopathy are considered at risk of cardiac arrest on induction of anesthesia [1], and patients with dilated cardiomyopathy or acute myocardial infarction are extremely susceptible to the cardiodepressive effect of anesthetics under general anesthesia [2]. In case 1, the patient had a low left ventricular ejection fraction (20-25\%) due to heart failure resulting from dilated cardiomyopathy, and in case 2 , the patient revealed unstable hemodynamics due to myocardial infarction and septic shock. In both patients', general conditions were life threatening and coagulation profiles were abnormal. Therefore, we chose PNBs for anesthesia instead of general anesthesia or a central neuraxial block.

PNBs provide more stable intraoperative hemodynamics than general anesthesia or a central neuraxial block $[3,4]$. Ultrasound-guided combined femoral and sciatic nerve blocks are mainly chosen for lower limb surgery. PNB has the strong advantage of hemodynamic stability, which has been reported to be related to increased survival. Reported death rates after lower limb amputation range from 8 to $30 \%$, but reports indicate PNB has a lower mortality rate in patients with high risk cardiac diseases than neuraxial block $[2,3,5]$. In our cases, PNBs were safely performed for lower limb surgery in patients with high risk cardiac disease. 


\section{References}

1. Lynch J, Pehora C, Holtby H, Schwarz SM, Taylor K. Cardiac arrest upon induction of anesthesia in children with cardiomyopathy: an analysis of incidence and risk factors. Paediatr Anaesth 2011; 21: 951-7.

2. Honda A, Arai T, Akiyama M, Masuda E, Kobayashi M, Hoka S. Anesthetic management of a patient with a history of Batista procedure for dilated cardiomyopathy undergoing gastric surgery. J Anesth 2006; 20: 227-30.

3. Bech B, Melchiors J, Børglum J, Jensen K. The successful use of peripheral nerve blocks for femoral amputation. Acta Anaesthesiol Scand 2009; 53: 257-60.

4. Chia N, Low TC, Poon KH. Peripheral nerve blocks for lower limb surgery-a choice anaesthetic technique for patients with a recent myocardial infarction? Singapore Med J 2002; 43: 583-6.

5. Kocum A, Turkoz A, Bozdogan N, Caliskan E, Eker EH, Arslan G. Femoral and sciatic nerve block with 0.25\% bupivacaine for surgical management of diabetic foot syndrome: an anesthetic technique for high-risk patients with diabetic nephropathy. J Clin Anesth 2010; 22: 363-6. 